\title{
A New Frequency Model for Pump-to-Signal RIN Transfer in Brillouin Fiber Amplifiers
}

\author{
Junhe Zhou, Jianping Chen, Yves Jaouën, Lilin Yi, Xinwan Li, Hervé Petit, and Philippe Gallion
}

\begin{abstract}
In this letter, we propose a novel frequency model to evaluate the pump-to-signal relative intensity noise (RIN) transfer in stimulated Brillouin scattering (SBS). For the first time, the RIN transfer in Brillouin fiber amplifiers has been numerically determined in the pump depletion regime both in the time and frequency domains. Moreover, an analytical expression of RIN transfer has been derived from the well-known SBS coupling equations when pump depletion is ignored. Experimental results are found to be in good agreement with the theoretical analysis.
\end{abstract}

Index Terms-Amplifier noise, Brillouin scattering, optical fiber.

\section{INTRODUCTION}

$\mathbf{T}$ HE stimulated Brillouin scattering (SBS) process in optical fibers is an attractive phenomenon. It can be applied in the case of Brillouin lasers [1], [2], optical sensors, or narrow bandpass filters [3]. Furthermore, Brillouin fiber amplifiers can induce slow light, which has been paid significant attention to recently [4]. Pump-to-signal relative intensity noise (RIN) transfer in Raman amplification [5] and parametric amplification [6] has been thoroughly studied. RIN transfer is weak at high frequency, especially for the counterpumping scheme. In the SBS process, the pump wave counterpropagates with the signal wave. However, the Brillouin gain coefficient is three orders larger than the Raman one and requires shorter fiber as the gain medium, which results in the reduction of the walkoff effect. Moreover, since the pump is usually broadened to achieve wider amplification bandwidth [7], the RIN transfer spectrum will also be broadened. The RIN transfer has been investigated in the case of Brillouin fiber lasers [1], [2]. However, no complete expression of RIN transfer function has been proposed in the case of Brillouin fiber amplifiers.

Theoretical studies on the dynamics and the noise of Brillouin lasers are usually performed in the time domain because the fiber lengths are generally short [1]. However, the method is very computer-time-consuming if the fiber length is long (which is usually the case in SBS induced slow light experiments) and the pump modulation frequency is high. In this letter, we propose a frequency model, which is based on perturbation theory, to study the pump-to-signal RIN transfer in Brillouin amplifiers.

Manuscript received February 6, 2007; revised March 22, 2007.

J. Zhou and L. Yi are with École Nationale Supérieure des Télécommunications (GET/Télécom Paris and CNRS UMR 5141LTCI), Paris 75013, France, and also with Shanghai Jiaotong Univerisity, Shanghai 200240, China (e-mail: junhe.zhou@enst.fr).

J. Chen and X. Li are with Shanghai Jiaotong University, Shanghai, 200240, China (e-mail: jpchen62@sjtu.edu.cn).

Y. Jaouën, H. Petit, and P. Gallion are with École Nationale Supérieure des Télécommunications and CNRS UMR5141, Paris 75013, France (e-mail: yves. jaouen@enst.fr).

Color versions of one or more of the figures in this letter are available online at http://ieeexplore.ieee.org.

Digital Object Identifier 10.1109/LPT.2007.898756
Comparison between the results obtained by the classical time domain model and our method illustrates its validity and efficiency. Analytical expressions can be derived from this model neglecting the pump depletion. Detailed numerical simulations are provided along with experimental measurements afterwards.

\section{Mathematical Modeling}

The amplitudes of the pump and the Stokes waves obey the following equations during the propagation in fibers [8]:

$$
\begin{aligned}
-\frac{\partial E_{p}}{\partial z}+\frac{n_{g}}{c} \frac{\partial E_{p}}{\partial t} & =\frac{-\alpha}{2} E_{p}-\frac{g_{B}}{2 A} E_{s} Q \\
\frac{\partial E_{s}}{\partial z}+\frac{n_{g}}{c} \frac{\partial E_{s}}{\partial t} & =-\frac{\alpha}{2} E_{s}+\frac{g_{B}}{2 A} E_{p} Q^{*} \\
\frac{\partial Q}{\partial t}+\left(\frac{\Gamma_{B}}{2}-i \Delta \omega\right) Q & =\frac{\Gamma_{B}}{2} E_{P} E_{s}^{*}
\end{aligned}
$$

where $E_{p}$ and $E_{s}$ are the amplitudes of the pump and the Stokes waves, $Q$ is the amplitude of the acoustic wave, $g_{B}$ is the Brillouin peak gain coefficient, $\Delta \omega$ is the detuning of the Stokes wave from the Brillouin peak gain wavelength, $A$ is the effective area, $\Gamma_{p}$ is the phonon decay rate, $\alpha g$ is the attenuation coefficient, and $n_{g}$ is the group refractive index.

We treat the noise term on the pump as a small perturbation and the resulting amplitude fluctuations are denoted as $\Delta E_{p}(z, t), \Delta E_{s}(z, t)$, and $\Delta Q(z, t)$. The fluctuations are small as compared with the corresponding steady state amplitudes $E_{p}(z), E_{s}(z)$, and $Q(z)$. Substituting them into (1) and taking the Fourier transform on the both sides, we have

$$
\begin{aligned}
-\frac{\partial \Delta \tilde{E}_{p}}{\partial z}-i \omega \frac{n_{g}}{c} \Delta \tilde{E}_{p}= & -\frac{\alpha}{2} \Delta \tilde{E}_{p}-\frac{g_{B}}{2 A} \Delta \tilde{E}_{s} Q \\
& -\frac{g_{B}}{2 A} \tilde{E}_{s} \Delta \tilde{Q} \\
\frac{\partial \Delta \tilde{E}_{s}}{\partial z}-i \omega \frac{n_{g}}{c} \Delta \tilde{E}_{s}= & -\frac{\alpha}{2} \Delta \tilde{E}_{s}+\frac{g_{B}}{2 A} \Delta \tilde{E}_{p} Q^{*} \\
& +\frac{g_{B}}{2 A} E_{p} \Delta \tilde{Q}^{*} \\
\Delta \tilde{Q}= & \frac{\frac{\Gamma_{B}}{2} \Delta \tilde{E}_{P} E_{s}^{*}+\frac{\Gamma_{B}}{2} E_{P} \Delta \tilde{E}_{s}^{*}}{\frac{\Gamma_{B}}{2}-i(\omega+\Delta \omega)}
\end{aligned}
$$

where $\Delta \tilde{E}(z, \omega)$ and $\Delta \tilde{E}^{*}(z,-\omega)$ are the Fourier transformations of $\Delta E(z, t)$ and $\Delta E^{*}(z, t)$. Equation (2) is a set of ordinary differential equations. It can be integrated by the numerical methods such as the Runge-Kutta method.

We use typical parameters of a nonzero dispersion-shifted fiber for modeling: $\mathrm{A}=50 \mu \mathrm{m}^{2}, \mathrm{~g}_{\mathrm{B}}=5 \times 10^{-11} \mathrm{~m} / \mathrm{W}$, and $\Gamma_{\mathrm{B}} / 2 \pi=40 \mathrm{MHz}$. For the sake of simplicity, the 

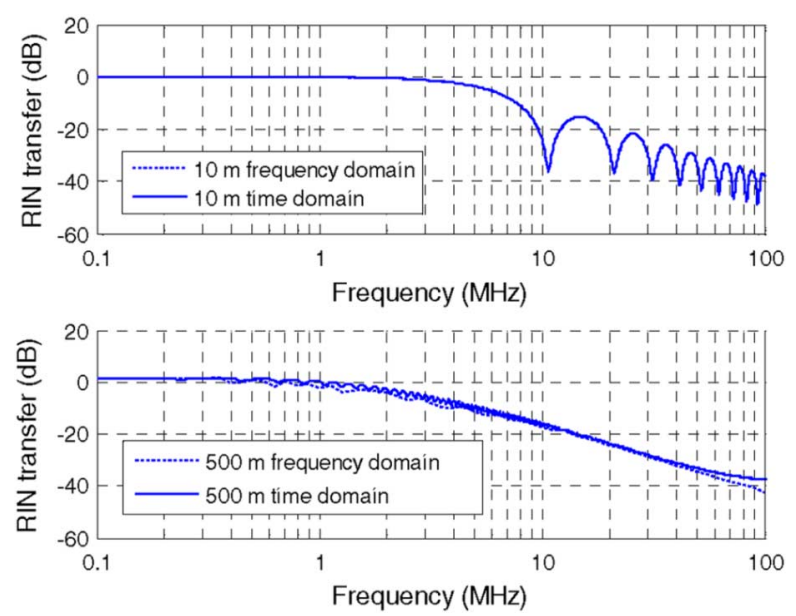

Fig. 1. Comparison of RIN transfer with different fiber length obtained by temporal and frequency methods (the pump power is $100 \mathrm{~mW}$ ).

signal detuning from the gain peak $\Delta \omega$ is 0 . The pump RIN $r_{p}=(\Delta P / P)^{2}$ is fixed to $-100 \mathrm{~dB}$, and the input signal power is $-20 \mathrm{dBm}$. The RIN transfer function is defined as $10 \log \left(r_{s} / r_{p}\right)$.

Fig. 1 presents comparison of RIN transfer based on the temporal and the frequency models for 10- and 500-m fiber. The pump power is $100 \mathrm{~mW}$. The results show very good agreement, especially in the case of 10-m fiber, whose curves are identical. The numerical solutions of both temporal and frequency models take into account pump depletion. Calculation of RIN transfer functions requires about $6 \mathrm{~h}$ in the time domain for the 500-m fiber, whereas only $2 \mathrm{~min}$ in the frequency domain. The efficiency is caused by the transformation of the two-dimensional partial differential equations into one-dimensional ordinary differential equations. Fig. 2 displays the results of RIN transfer with different pump powers with the 50-m fiber. The results show that when the gain is not saturated, the RIN transfer increases with pump power, which is consistent with the analytical expression presented in Section III. When the gain is fully saturated, the RIN transfer experiences reduction due to the saturation of the pump power.

\section{Analytical Solution for the SMall Signal CaSe}

By substituting $\Delta \tilde{Q}$ expression in the second equation of (2), and after some straightforward mathematical calculations, the signal amplitude fluctuation $\Delta \tilde{E}_{s}$ is expressed as

$$
\begin{aligned}
\frac{\partial \Delta \tilde{E}_{s}}{\partial z}=\left(i \omega \frac{n_{g}}{c}-\frac{\alpha}{2}+k\left|E_{p}\right|^{2}\right) \Delta \tilde{E}_{s} & \\
& +k E_{s}\left(\frac{k^{\prime}}{k} \Delta \tilde{E}_{p} E_{P}^{*}+E_{p} \Delta \tilde{E}_{p}^{*}\right)
\end{aligned}
$$

where

$$
\begin{aligned}
k & =\frac{g_{B}}{2 A} \frac{\frac{\Gamma_{B}}{2}}{\frac{\Gamma_{B}}{2}+i(\omega+\Delta \omega)} \\
k^{\prime} & =\frac{g_{B}}{2 A} \frac{\frac{\Gamma_{B}}{2}}{\frac{\Gamma_{B}}{2}+i \Delta \omega} .
\end{aligned}
$$

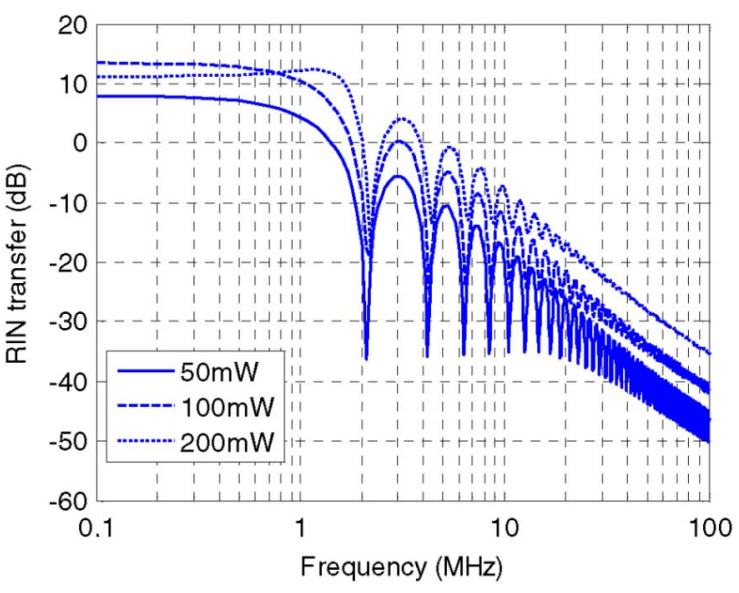

Fig. 2. Theoretical RIN transfer function for different pump powers (the fiber length is $50 \mathrm{~m}$ ).

Solving (3) gives the following analytical expression:

$$
\begin{aligned}
& \Delta \tilde{E}_{s}(L) \\
& =\exp \left(\int_{0}^{L}\left(i \omega \frac{n_{g}}{c}-\frac{\alpha}{2}+k\left|E_{p}\right|^{2}\right) d z\right) \\
& \quad * \int_{0}^{L} k E_{s}\left(\frac{k^{\prime}}{k} \Delta \tilde{E}_{p} E_{P}^{*}+E_{p} \Delta \tilde{E}_{p}^{*}\right) \\
& \quad \times \exp \left(-\int_{0}^{z}\left(i \omega \frac{n_{f g}}{c}-\frac{\alpha}{2}+k\left|E_{p}\right|^{2}\right) d l\right) d z
\end{aligned}
$$

where $L$ is the fiber length, and $z$ is the distance from the local fiber point to the fiber input. Neglecting the pump depletion, (4) can be expressed as

$$
\begin{aligned}
\frac{\Delta \tilde{E}_{s}(L)}{E_{s}(L)}= & k G(L) \int_{0}^{L}\left(\frac{k^{\prime}}{k} \Delta \tilde{E}_{p} E_{P}^{*}+E_{p} \Delta \tilde{E}_{p}^{*}\right) \\
& \times G(z)^{-1} \exp \left(i \omega \frac{n_{g}}{c}(L-z)\right) d z \\
G(z)= & \exp \left(\left(k-k^{\prime}\right) P_{p} Z_{\text {eff }}\right)
\end{aligned}
$$

where

$$
Z_{\text {eff }}=\frac{1-\exp (-\alpha z)}{\alpha} .
$$

Equation (5) can be used to derive the RIN transfer function $10 \log \left(r_{s} / r_{p}\right)$. It can be further simplified at low frequency (i.e., for $\omega=0 G(z)=1$ ) as

$$
\begin{aligned}
\frac{\Delta \tilde{E}_{s}(L)}{E_{s}(L)} & =\frac{g_{B}}{2 A} \frac{\frac{\Gamma_{B}}{2}}{\frac{\Gamma_{B}}{2}+i \Delta \omega} \Delta \tilde{P}(L, 0) L_{\mathrm{eff}} \\
\Delta \tilde{P}(L, 0) & =\Delta \tilde{E}_{p}(L, 0) E_{P}^{*}(L, 0)+E_{p}(L, 0) \Delta \tilde{E}_{p}^{*}(L, 0) .
\end{aligned}
$$

Finally, the signal RIN $r_{s}$ is expressed as

$$
r_{s}=\left(\frac{\Delta \tilde{P}_{s}}{P_{s}}\right)^{2}=r_{p}\left(\frac{\frac{g_{B}}{A}}{1+\left(\Delta \omega / \frac{\Gamma_{B}}{2}\right)^{2}} P_{p} L_{\mathrm{eff}}\right)^{2} .
$$



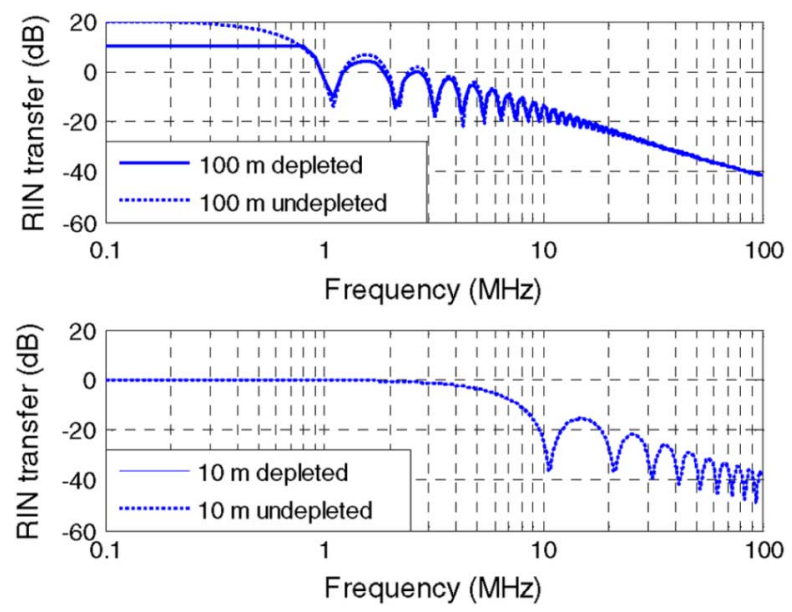

Fig. 3. Comparison of RIN transfer between the numerical (depleted) and analytical (undepleted) results (the pump power is $100 \mathrm{~mW}$ ).

In Fig. 3, the numerical and the analytical results are compared. The pump power is fixed to $100 \mathrm{~mW}$ and the fiber lengths are 10 and $100 \mathrm{~m}$, respectively. The analytical expression gives results in excellent agreement with the numerical ones in the case of the 10-m fiber. In such short fiber, the pump is not depleted, so the analytical and numerical RIN transfer functions are exactly identical. Also, the fiber length can be viewed as the effective length. From (7), the RIN transfer at low frequency can be directly calculated, which agrees well with the numerical model. When the fiber is longer and the pump is depleted, the different curves show an accuracy reduction, i.e., (5) and (7) are not valid in the pump depletion regime. Gain saturation will cause the reduction of RIN transfer. However, in the broadened pump configuration, the pump power is distributed over a wide spectrum. So, the pump also works in the undepleted regime, where (5) and (7) are valid. The figures also show that counterpropagation averages the noise over the fiber, creating the extinction of $20 \mathrm{~dB}$ per decade at high frequency, like an electrical low-pass filter. The cutoff frequency results from the Brillouin gain and the walkoff effect. The dip frequency shown in Fig. 3 can be calculated using the formula (40) in [5].

\section{EXPERIMENTAL MEASUREMENT}

We measure the RIN transfer in an SBS amplifier with a monochromatic pump modulated with sinusoidal wave. A 300-m Truewave fiber is used as the gain medium. The pump laser diode at $1548 \mathrm{~nm}$ is modulated using a Mach-Zehnder modulator driven by the electrical sinusoidal wave generator in a network analyzer (HP $4194 \mathrm{~A}$ ranging $0.1 \mathrm{~Hz}-100 \mathrm{MHz}$ ). It is then amplified by a high-power erbium-doped fiber amplifier. The pump power injected into the fiber is $14 \mathrm{dBm}$. A stable tunable laser source with the power of $-20 \mathrm{dBm}$ is used as the signal detuning from the pump wavelength for $10.764 \mathrm{GHz}$. An optical circulator is placed at the fiber input to act as an isolator for the injected signal. A second optical circulator is placed at the fiber output to extract the signal that counterpropagates with the pump. Two polarization controllers are placed after the pump and signal laser sources to maximize the output power. The output signal is detected by a photodetector and the RIN transfer function is measured using the network analyzer.

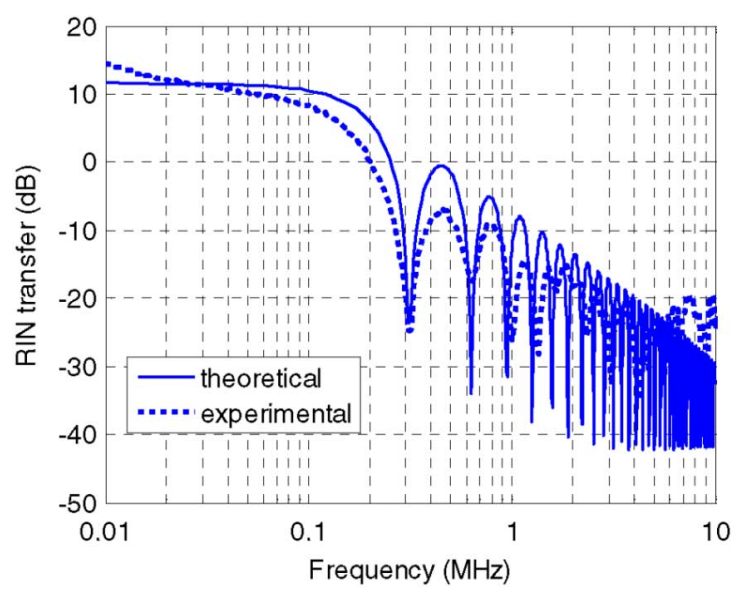

Fig. 4. Comparison of theoretical and measured RIN transfer (the fiber length is $300 \mathrm{~m}$, and the pump power is $14 \mathrm{dBm}$ ).

The experimental results are compared with the theoretical predictions in Fig. 4. The two curves show nice agreement and the discrepancy might result from the transfer function of the modulator.

\section{CONCLUSION}

A novel efficient frequency model capable of deriving the RIN transfer in the pump depletion regime has been proposed to investigate the pump-to-signal RIN transfer in SBS. Analytical expressions have been derived under undepleted pump assumption. Detailed numerical simulations have been provided afterwards, demonstrating the efficiency and validity of the model. Experimental measurements have been performed to further verify the theoretical predictions. Moreover, using the derived analytical expressions and separating the pump source into many discrete pumps, we will be able to characterize the RIN transfer in the SBS process with broadband pump and signal. The theoretical and experimental work on RIN transfer in the SBS process with a broadened pump is under way.

\section{REFERENCES}

[1] L. Stepien, S. Randoux, and J. Zemmouri, "Intensity noise in Brillouin fiber ring laser," J. Opt. Soc. Amer., vol. B19, no. 5, pp. 1055-1066, May 2002.

[2] J. Geng and S. Jiang, "Pump-to-stokes transfer of relative intensity noise in Brillouin fiber ring lasers," Opt. Lett., vol. 32, pp. 11-13, 2007.

[3] T. Tanemura, Y. Takushima, and K. Kikuchi, "Narrowband optical filter, with a variable transmission spectrum, using stimulated Brillouin scattering in optical fiber," Opt. Lett., vol. 27, pp. 1552-1554, 2002.

[4] Z. Zhu, "Numerical study of all-optical slow-light delays via stimulated Brillouin scattering in an optical fiber," J. Opt. Soc. Amer. B, vol. 22, pp. 2378-2384, 2005.

[5] C. R. S. Fludger, V. Handerek, and R. J. Mears, "Pump to signal RIN transfer in Raman fiber amplifiers," J. Lightw. Technol., vol. 19, no. 8, pp. 1140-1148, Aug. 2001.

[6] M. E. Marhic, G. Kalogerakis, K. K. Y. Wong, and L. G. Kazovsky, "Pump-to-signal transfer of low-frequency intensity modulation in fiber optical parametric amplifiers," J. Lightw. Technol., vol. 23, no. 3, pp. 1049-1055, Mar. 2005.

[7] Z. Zhu, A. M. C. Dawes, D. J. Gauthier, L. Zhang, and A. E. Willner, "12-GHz-bandwidth SBS slow light in optical fibers," presented at the The Optical Fiber Communications Conf., Anaheim, CA, 2006, Paper PDP1.

[8] G. P. Agrawal, Nonlinear Fiber Optics, 3rd ed. New York: Academic, 2001. 